\title{
Beetroot juice attenuates glycaemic response in healthy volunteers
}

\author{
P.Y. Chang, M.S. Hafiz and C. Boesch \\ School of Food Science and Nutrition, Nutrition and Public Health Group, University of Leeds, LS2 9JT, Leeds, UK.
}

The consumption of red beetroot has been associated with a number of health benefits. Antidiabetic properties have been suggested in the literature, yet evidence from in vivo and in vitro studies is very limited ${ }^{(1)}$. A recent study has indicated a moderate reduction of postprandial glucose and insulin response following neobetanin rich beetroot juice consumption ${ }^{(2)}$. Reductions of both, glucose and insulin, are considered beneficial with regards to lowering the risk of type 2 diabetes ${ }^{(3)}$. The aim of our study was to investigate the acute effects of beetroot juice consumption in combination with carbohydrates on the glycaemic response in humans.

We recruited ten healthy volunteers ( 1 male and 9 females), aged 20-24 years and BMI ranging from $16 \cdot 7-26 \cdot 8$, for a randomized crossover study. Following an overnight fast, participants were asked to consume $270 \mathrm{ml}$ beetroot juice or a sugar-matched control drink alongside white bread to provide $50 \mathrm{~g}$ available carbohydrates in each setting. The sugar content in the juice was analysed using HPLC prior to study start (sucrose $79.9 \%$, glucose $10.9 \%$, fructose $9.4 \%$ ). Capillary blood was collected via finger prick in regular intervals following juice and bread consumption $(0,15,30,45,60,90,120,150,180 \mathrm{~min})$ and blood glucose levels determined using glucometer. Data were analysed using two-tailed paired t-test.

The consumption of beetroot juice in comparison to the sugar matched control drink reduced blood glucose levels significantly after 15, 30, 90 and 180 min (Fig 1, p < 0.05), demonstrating a reduction in peak and early phase as well as a delay in glycaemic response. All individuals responded to beetroot juice with a reduced or delayed blood glucose, however, to a differing extent which was not related to body weight or BMI.

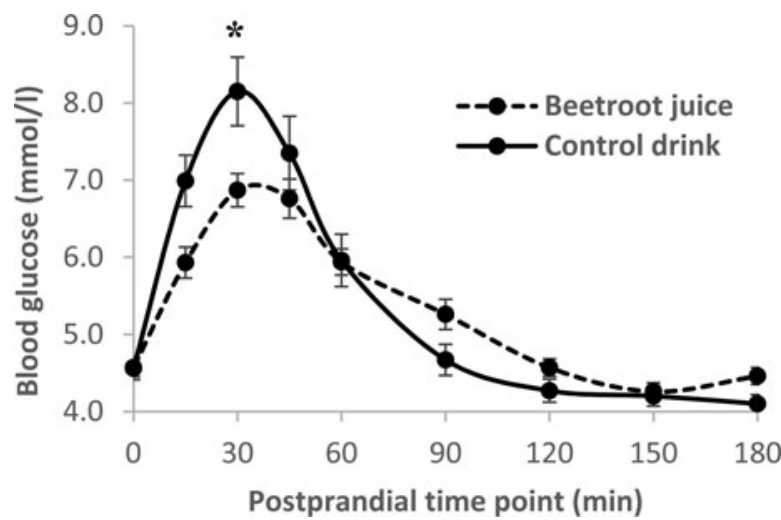

Fig. 1. Blood glucose concentration at baseline and following postprandial phase over $180 \mathrm{~min}$. Values represent mean with SE of ten participants. * indicate significant differences $(\mathrm{p}<0.05 ; \mathrm{t}$-test $)$.

The lower blood glucose peak as well as a delayed glucose appearance indicate potential beneficial effects of beetroot juice consumption on postprandial glucose response which may relate to different mechanisms such as the inhibition of digestive enzymes, reduced and/or delayed glucose uptake, mechanisms that may involve betalain pigments, polyphenols as well as nitrates found in beet.

This work was funded by the Gen Foundation and the N8AgriFood consortium.

1. Clifford T, Howatson G, West DJ \& Stevenson EJ (2015) Nutrients 7, 2801-2822.

2. Wootton-Beard PC, Brandt K, et al. (2014) J Nutr Sci 3, pe9.

3. Coe S \& Ryan L (2016) J Nutr Sci 5, pe2410. 